\title{
Transport properties of a quasi-two-dimensional electron system formed in $\mathrm{LaAlO}_{3} / \mathrm{EuTiO}_{3} / \mathrm{SrTiO}_{3}$ heterostructures
}

\author{
G. M. De Luca, ${ }^{*}$ R. Di Capua ${ }^{\dagger}$ E. Di Gennaro, F. Miletto Granozio, D. Stornaiuolo, and M. Salluzzo \\ CNR-SPIN and Dipartimento di Fisica Università “Federico II,” Complesso Monte Santangelo via Cinthia, I-80126 Napoli, Italy
}

A. Gadaleta, I. Pallecchi, and D. Marrè

CNR-SPIN and Dipartimento di Fisica, Università di Genova, Via Dodecaneso 33, I-14146 Genova, Italy

C. Piamonteze

Swiss Light Source, Paul Scherrer Institut, CH-5232 Villigen PSI, Switzerland

M. Radovic

Swiss Light Source and SwissFEL, Paul Scherrer Institut, CH-5232 Villigen PSI, Switzerland

Z. Ristic and S. Rusponi

Institute of Condensed Matter Physics, Ecole Polytechnique Fédérale de Lausanne, CH-1015 Lausanne, Switzerland

(Received 26 March 2014; revised manuscript received 30 May 2014; published 23 June 2014)

\begin{abstract}
We study the structural, magnetic, and transport properties of $\mathrm{LaAlO}_{3} / \mathrm{EuTiO}_{3} / \mathrm{SrTiO}_{3}$ heterostructures grown by pulsed laser deposition. The samples are characterized in situ by electron diffraction and scanning probe microscopy and ex situ by transport measurements and x-ray absorption spectroscopy. $\mathrm{LaAlO}_{3} / \mathrm{EuTiO}_{3} / \mathrm{SrTiO}_{3}$ films show a ferromagnetic transition at $T \leqslant 7.5 \mathrm{~K}$, related to the ordering of $\mathrm{Eu}^{2+}$ spins, even in samples characterized by just two $\mathrm{EuTiO}_{3}$ unit cells. A finite metallic conductivity is observed only in the case of samples composed by one or two $\mathrm{EuTiO}_{3}$ unit cells and, simultaneously, by a $\mathrm{LaAlO}_{3}$ thickness greater than or equal to four unit cells. The effect of ferromagnetic $\mathrm{EuTiO}_{3}$ on the transport properties of $\delta$-doped $\mathrm{LaAlO}_{3} / \mathrm{EuTiO}_{3} / \mathrm{SrTiO}_{3}$ is critically discussed.
\end{abstract}

DOI: 10.1103/PhysRevB.89.224413

PACS number(s): 75.70.-i

\section{INTRODUCTION}

The discovery of a quasi-two-dimensional electron system (Q2DES) at the interface between $\mathrm{LaAlO}_{3}(\mathrm{LAO})$ and $\mathrm{SrTiO}_{3}$ (STO) insulators [1] stimulated intense research on novel functional oxide heterostructures. In particular, the intriguing electronic properties of the Q2DES in the LAO/STO system have generated an intense debate about the physics of oxide interfaces. Among the most interesting characteristics of the Q2DES, we cite the transport dominated by a remarkably large Rashba spin-orbit coupling [2] and low-temperature two-dimensional superconductivity (SC). Both phenomena can be tuned by using the electric field effect. In particular, a metal (SC at low T)-to-insulating transition can be obtained even at room temperature $[3,4]$. At the same time, different reports suggest the presence of some magnetic order, possibly coexisting with SC [5-7]. In view of these unique properties, the integration of LAO/STO into modern electronics, in particular, spintronics, is an exciting challenge. The recent reports of resonant amplification [8] and of long lifetimes [9] of spin-polarized electrons injected in STO heterostructures are encouraging in these directions. On the other hand, the realization of a spin-polarized Q2DES at the equilibrium remains at the moment only an ambitious target. Indeed, the interpretation of the experimental signatures of magnetism in $\mathrm{LAO} / \mathrm{STO}$ heterostructures remains controversial, and more

\footnotetext{
*gabriella.deluca@spin.cnr.it

†rdicapua@na.infn.it
}

recent reports suggest a spin polarization of the Q2DES only in the presence of oxygen vacancies [10].

A promising route to obtain a homogeneous and robust spin polarization at equilibrium in LAO/STO is by $\delta$ doping with a magnetic layer. Yet the first attempts at $\delta$ doping have shown that in general it induces charge localization $[11,12]$, and in the case of insertion of rare-earth oxides, it gives rise to electron correlation effects possibly enhanced by structural distortions [13]. Here we show that $\mathrm{EuTiO}_{3}$ (ETO) can be used as magnetic $\delta$-doping layer to obtain heterostructures characterized by a Q2DES interacting with a ferromagnetic film. This result represents a first step toward the realization of an oxide spin-polarized Q2DES.

$\mathrm{EuTiO}_{3}$ is a perovskite whose lattice parameters $(a=$ $b=c=3.905 \AA$ ) perfectly match those of STO, allowing strain-free epitaxial growth. Its electronic structure is also very similar to that of STO, with a broad band about $3.0 \mathrm{eV}$ below the Fermi level originating from O-2 $p$ states and a conduction band formed by Ti-3 $d$ states. However, ETO possesses an additional narrow Eu- $4 f$ band, lying just below the Fermi level, located within a direct band gap of $\sim 0.93 \mathrm{eV}$, much smaller than those of STO $(\sim 3.2 \mathrm{eV})$ and LAO $(\sim 5.6 \mathrm{eV})$ [14]. Bulk ETO has a G-type antiferromagnetic ground state below the Néel temperature $\mathrm{T}_{N} \sim 5.5 \mathrm{~K}$, which is switched to a ferromagnetic state by doping [15] or lattice strain [16]. The lattice, electronic, and magnetic properties of ETO, as well as the nonpolar character of the (001) surface, similar to STO, make it a perfect candidate as a magnetic dopant layer for LAO/STO interfaces. 


\section{SAMPLE FABRICATION}

We deposited $\operatorname{LAO}(n) / \operatorname{ETO}(m) / \mathrm{STO}[n=0-12$ unit cells (uc), and $m=0-5 \mathrm{uc}$ ] epitaxial heterostructures by RHEED (reflection high-energy electron diffraction)-assisted pulsed laser deposition (PLD) from sintered $\mathrm{Eu}_{2} \mathrm{Ti}_{2} \mathrm{O}_{7}$ and crystalline LAO targets onto $\mathrm{TiO}_{2}$-terminated (001) STO substrates. A $\mathrm{KrF}$ excimer laser (wavelength, $248 \mathrm{~nm}$; pulse rate, $1 \mathrm{~Hz}$ ) was focused on the target at a fluence of $1.3 \mathrm{~J} / \mathrm{cm}^{2}$. To prevent the formation of the competing $\mathrm{Eu}_{2} \mathrm{Ti}_{2} \mathrm{O}_{7}$ phase, ETO deposition must be carried out at an oxygen partial pressure $\left(p_{\mathrm{O}_{2}}\right)$ and deposition temperature $\left(T_{d}\right)$ lower than those usually employed for LAO/STO heterostructures. We adopted two sets of deposition parameters: set $\mathrm{A}-T_{d}=650-700^{\circ} \mathrm{C}$ and $p_{\mathrm{O}_{2}}=1 \times 10^{-2} \mathrm{~Pa}$ for both ETO and LAO films; and set B$T_{d}=600^{\circ} \mathrm{C}$ and $p_{\mathrm{O}_{2}}=1 \times 10^{-5} \mathrm{~Pa}$ for growth of ETO and $T_{d}=800^{\circ} \mathrm{C}$ and $p_{\mathrm{O}_{2}}=1 \times 10^{-2} \mathrm{~Pa}$ for LAO. No annealing procedures were carried out after the deposition to avoid recrystallization of ETO to the $\mathrm{Eu}_{2} \mathrm{Ti}_{2} \mathrm{O}_{7}$ phase at higher oxygen pressures. The samples were, in both cases, slowly cooled down to room temperature, at a rate of $3^{\circ} \mathrm{C} / \mathrm{min}$, in $10^{-2} \mathrm{~Pa}$ of $\mathrm{O}_{2}$.

\section{EXPERIMENTAL RESULTS}

In situ structural and surface characterizations were performed by RHEED (STAIB high-pressure RHEED) and scanning probe microscopy (Omicron VT-AFM) in the Modular facility for Oxide Deposition and Analysis (MODA) system. Chemical, electronic, and magnetic characterizations were carried out by $\mathrm{Eu}-\mathrm{M}_{4,5}$ edge $\mathrm{x}$-ray absorption spectroscopy (XAS) and X-ray magnetic circular dichroism (XMCD) at the $X$-TREME beam line of the Swiss Light Source at the Paul Scherrer Institut [17]. Finally, transport characterizations were performed using a Heliox $3 \mathrm{He}$ refrigerator and a Quantum Design PPMS system for magnetotransport and Hall effect measurements in magnetic fields up to $9 \mathrm{~T}$.

In Fig. 1 we present a series of experimental characterizations of the LAO/ETO/STO heterostructures. The RHEED analysis shows that all the samples have layer-by-layer growth and that their structural quality is similar to that of LAO/STO films. Apart from a progressive decrease in the intensity, the RHEED pattern measured on the STO (001) single crystal just before the deposition [Fig. 1(b)] does not change substantially with the deposition of ETO [Fig. 1(c)] and LAO [Fig. 1(d)] layers, suggesting epitaxial growth and perfect matching between the lattice parameters of the films and those of the substrate. In particular, no additional diffracted spot, possibly related to the $\mathrm{Eu}_{2} \mathrm{Ti}_{2} \mathrm{O}_{7}$ phase, appears in the thickness range investigated (1-6 uc). It is worth noting that the epitaxial growth of pyrochlore $(F d 3 m$ space group and cubic cell parameter of $10.45 \AA$ ) and monoclinic $\mathrm{Eu}_{2} \mathrm{Ti}_{2} \mathrm{O}_{7}$ [18] on STO (001) is extremely unlikely due to the large cost in terms of lattice mismatch and surface energy.

Atomic force microscopy [Fig. 1(e)] shows that the LAO/ETO/STO morphology mimics those of the STO substrates on which they are deposited, with flat terraces separated by 1-uc-high step edges. Finally, XAS and XMCD spectra [Fig. 1(f)] demonstrate that $\mathrm{Eu}$ is in the $\mathrm{Eu}^{2+}$ oxidation state with some admixtures of $\mathrm{Eu}^{3+}(\leqslant 30 \%)$, which can form as a consequence of the oxidizing atmosphere during the
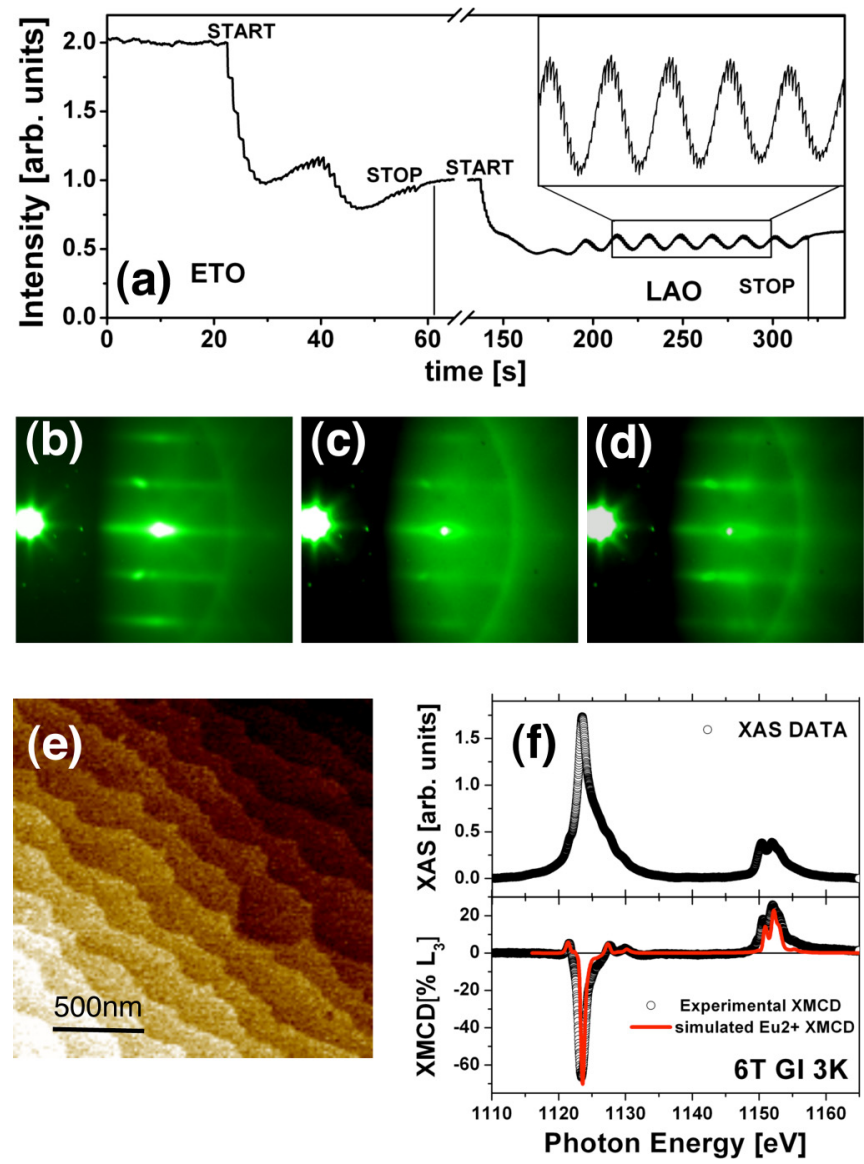

FIG. 1. (Color online) (a) Specular RHEED intensity vs deposition time during the growth of a LAO/ETO film on an STO single crystal. (b-d) RHEED pattern acquired (b) just before the deposition on STO, (c) after the deposition of ETO, and (d) after the deposition of LAO. (e) AFM topography on a conducting LAO(10 uc)/ETO(2 uc)/STO sample (set A). (f) Top: XAS sum spectra of circular polarized $(\mathrm{c}+$ and $\mathrm{c}-$ ) spectra at $3 \mathrm{~K}$ and $6 \mathrm{~T}$, with the field almost parallel to the interface (grazing incidence; GI). Bottom: Corresponding XMCD spectrum, which is the difference between the XAS data acquired with $\mathrm{c}+$ and $\mathrm{c}-$ circular polarizations $\left(I^{+}-I^{-}\right)$and corresponding simulated spectra (red line) using multiplet scattering theory for an $\mathrm{Eu}^{2+}$ ion.

deposition or due to partial Eu/La substitution. The XMCD signal, determined as the difference $\left(I^{+}-I^{-}\right)$between XAS spectra acquired with circular-plus $(\mathrm{c}+)$ and those acquired with circular-minus $(\mathrm{c}-$ ) polarizations, is perfectly reproduced using atomic scattering multiple calculations performed using the CTM4XAS code [19] and assuming uniquely the contribution of the majority of $\mathrm{Eu}^{2+}$ magnetic ions.

To determine the magnetic moment we employed the sum rules developed for rare-earth $4 f$ ions:

$$
\begin{aligned}
m_{\text {Espin }} & =\mu_{B} \frac{2 \int_{M_{5}}\left(I^{+}-I^{-}\right) d E-3 \int_{M_{4}}\left(I^{+}-I^{-}\right) d E}{2 \int_{M_{4}+M_{5}}\left(I^{+}+I^{-}\right)} 2 N_{4 f}, \\
m_{\mathrm{orb}} & =-\mu_{B} \frac{\int_{M_{5}}\left(I^{+}-I^{-}\right) d E+\int_{M_{4}}\left(I^{+}-I^{-}\right) d E}{\int_{M_{4}+M_{5}}\left(I^{+}+I^{-}\right)} 4 N_{4 f} .
\end{aligned}
$$


In Eqs. (1) and (2), the integrals are calculated around $M_{4}$, $M_{5}$, or $M_{4}+M_{5}$ (whole energy range) absorption edges, while $N_{4 f}$ is the number of holes in the $4 f$ states $\left(N_{4 f}=7\right.$ for $\left.\mathrm{Eu}^{2+}\right)$ and $m_{\text {Espin }}$ and $m_{\text {orb }}$ are the effective spin moment and orbital moment, respectively. The saturated spin moment, calculated using the sum rules [20], was found to be $3.5 \pm 0.2 \mu_{B} / \mathrm{Eu}$ in conducting LAO/ETO/STO and to increase slowly with the ETO thickness. This value is lower than that expected for $S_{z}=7 / 2 \mathrm{Eu}^{2+}$ ions of $7 \mu_{B} / \mathrm{Eu}$. The deviation could be related to the presence of nonmagnetic $\mathrm{Eu}^{3+}$ and to the extremely reduced ETO thickness in our heterostructures. In
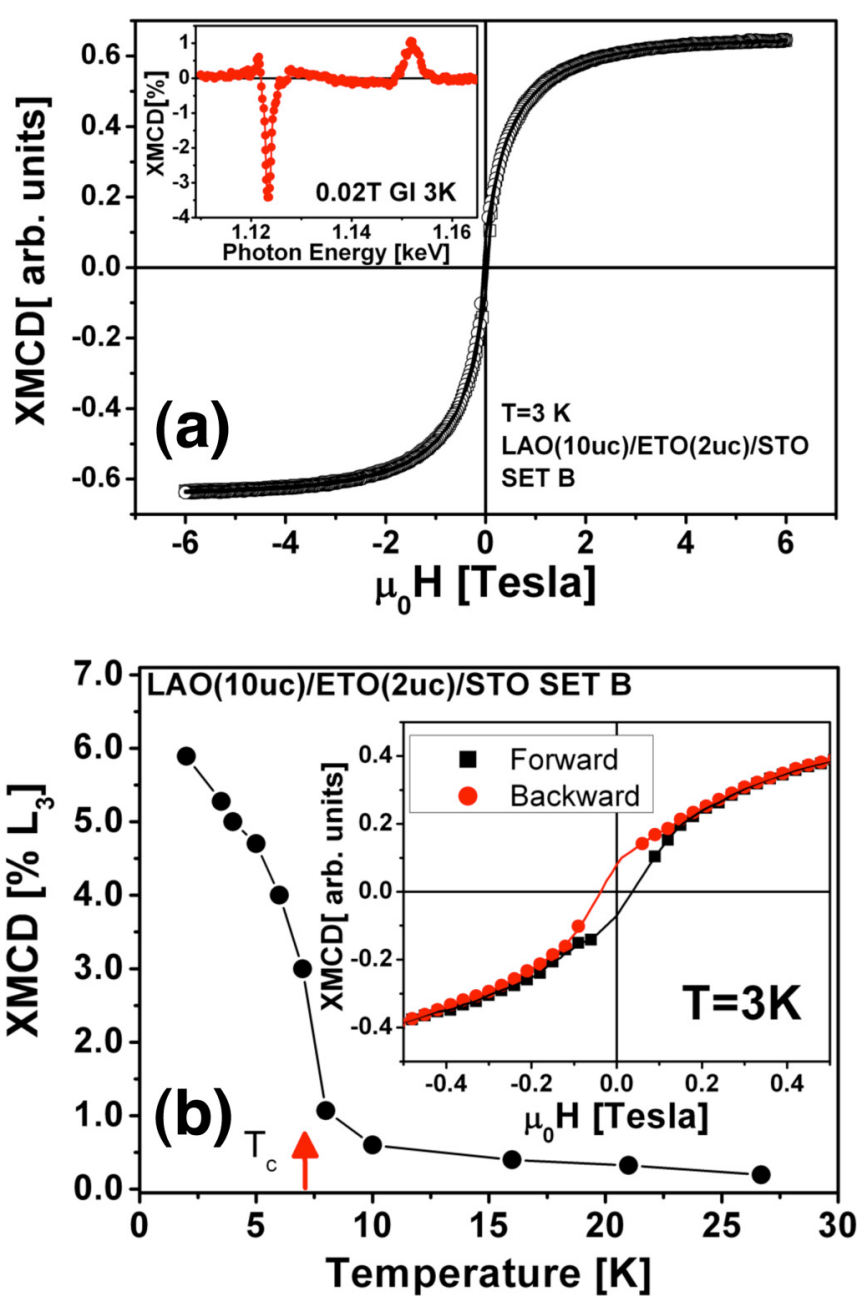

FIG. 2. (Color online) (a) Magnetic field dependence of the XMCD signal measured at the $M_{5}$ absorption edge at $3 \mathrm{~K}$ under grazing incidence conditions on a metallic LAO (10 uc)/ETO ( 2 uc)/STO heterostructure (sample set B). Inset: XMCD spectra at $0.02 \mathrm{~T}$ and $3 \mathrm{~K}$. (b) Temperature dependence of the XMCD intensity normalized to the $M_{5}$ intensity measured at $0.1 \mathrm{~T}$ after saturation of the magnetization at $6 \mathrm{~T}$ at low temperatures. The vertical (red) arrow indicates the ferromagnetic transition temperature. Inset: Low-magnetic-field region of the XMCD hysteresis loop showing the presence of a remanence at $3 \mathrm{~K}$. Filled symbols are experimental data, while solid lines are the same data extrapolated to the region around zero field using a cubic spline method [21]. Black squares and line, forward sweeping direction of the field; (red) circles and line, backward sweeping direction.
Fig. 2 we show a typical magnetic hysteresis loop at $3 \mathrm{~K}$ and a temperature dependence of the maximum of the XMCD signal of an LAO/ETO/STO heterostructure [21] characterized by an only 2-uc ETO $\delta$-doping layer embedded between a 10 -uc LAO film and the STO single crystal. The data show that thin ETO films are ferromagnetic, with the low field remanence of $0.3 \mu_{B} / \mathrm{Eu}$ [inset in Fig. 2(a)] [21]. Full alignment of the spin moments is achieved above $1 \mathrm{~T}$. The ferromagnetic transition temperature, measured at $0.1 \mathrm{~T}$, is about $7.5 \mathrm{~K}$. The switching from a bulk-like antiferromagnetic character to ferromagnetic behavior of the ETO layers could be related to electron doping and/or a slight oxygen excess as reported for films grown by pulsed laser deposition in an oxidizing atmosphere [22].

We now examine the electric transport behavior of these heterostructures. It is well known that LAO/STO interfaces become conducting for an LAO thickness equal to or larger than the critical value of $4 \mathrm{uc}$, which is usually explained in terms of the polar catastrophe mechanism. We expect that the nonpolar ETO does not alter the polarity stacking of the atomic layers. Consequently, we first explored the effect of the ETO thickness on the conductance of heterostructures
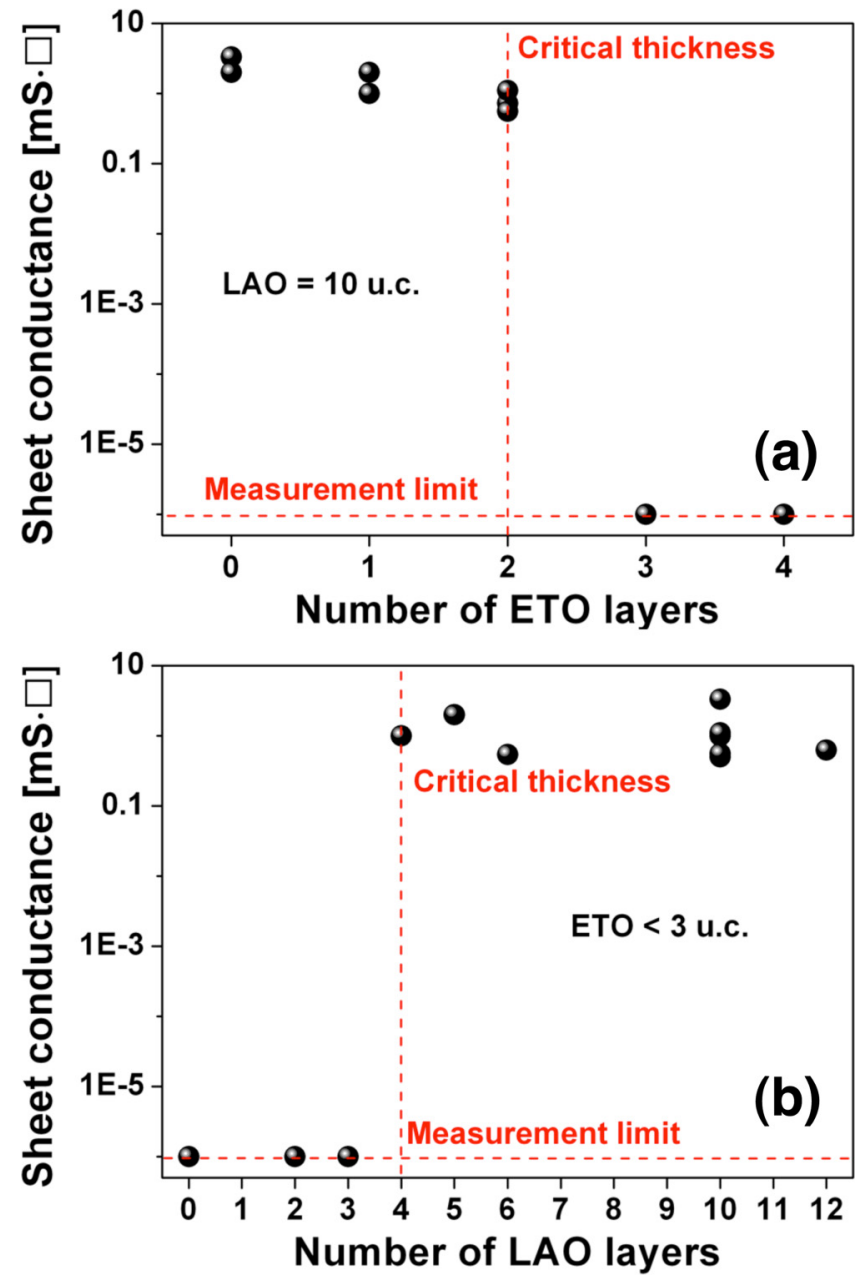

FIG. 3. (Color online) Sheet conductance of LAO/ETO/STO samples measured at room temperature (a) as a function of the number of ETO layers (LAO thickness $\geqslant 4 \mathrm{uc}$ ) and (b) as a function of the number of LAO layers (ETO thickness $\leqslant 3 \mathrm{uc}$ ). 
having a number of LAO layers larger than the critical value of $4 \mathrm{uc}$ (namely, $10 \mathrm{uc}$ ). We found that the number of ETO layers is a crucial parameter. As shown in Fig. 3(a), a finite conductance is present only for ETO films of 1 or $2 \mathrm{uc}$, whereas for higher thicknesses (up to $6 \mathrm{uc}$ ) the conductance of the interface is negligible in the limit of our experimental setup. On the other hand, by fixing the ETO thickness to 1 or $2 \mathrm{uc}$, we show in Fig. 3(b) that the conductance is finite only for LAO thicknesses equal to or larger than the critical value of $4 \mathrm{uc}$, as in the case of the usual LAO/STO interfaces. It is worth noting that STO/ETO/STO heterostructures, where a 10-uc STO film replaces LAO on top of ETO, are insulating, suggesting a crucial role of LAO in the creation of a metallic interface.

The sheet charge carrier density of LAO/ETO/STO heterostructures is similar to that of LAO/STO samples and ranges from $3 \times 10^{13}$ to $5 \times 10^{13}$ (set A) and from $8 \times$ $10^{13}$ to $10 \times 10^{13} e-/ \mathrm{cm}^{-2}$ (set $\mathrm{B}$ ) at low temperatures. The temperature dependence of the sheet resistance and of the mobility for some representative samples is shown in Figs. 4(a) and 4(b). The sheet resistance exhibits a metallic behavior, with values below $30 \mathrm{k} \Omega$ at $300 \mathrm{~K}$, typical of LAO/STO oxide interfaces. On the other hand, LAO/ETO/STO samples are characterized by a significant resistance upturn
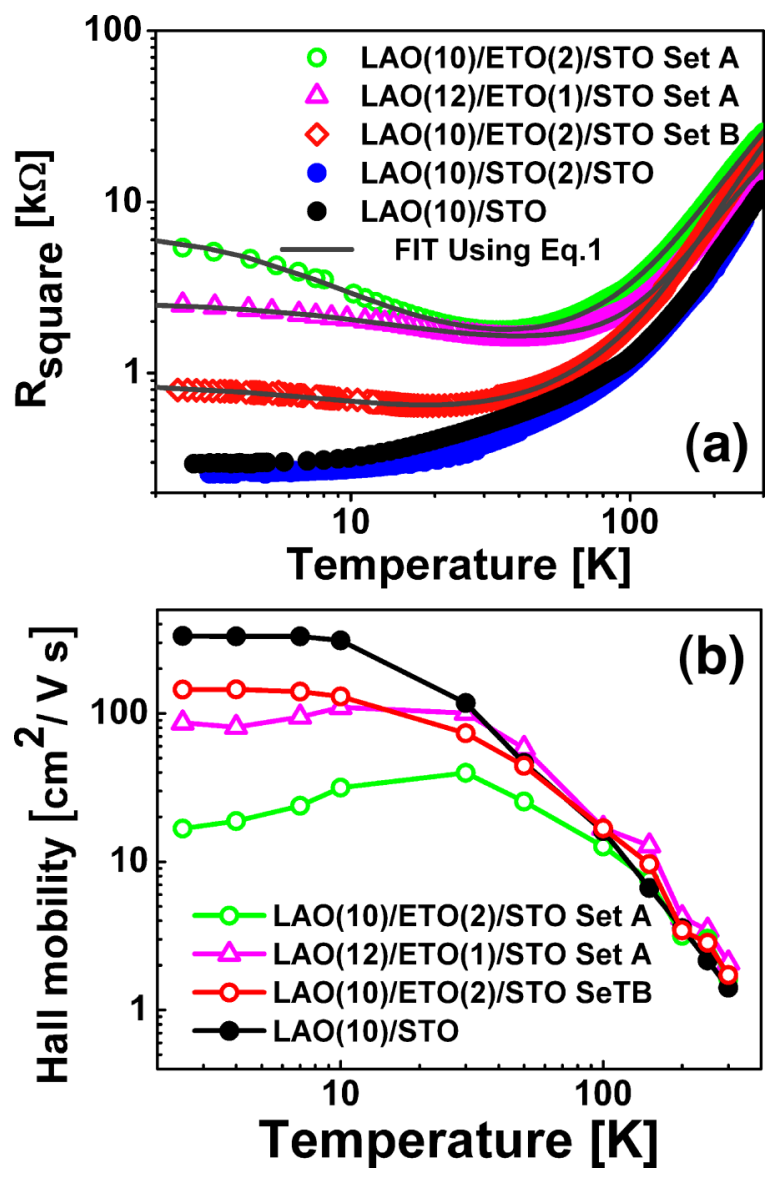

FIG. 4. (Color online) Temperature dependence of (a) the sheet resistance and (b) of the mobility of LAO/ETO/STO and LAO/STO heterostructures as labeled: Solid black lines in (a) are the fitting curves obtained using Eqs. (3) and (4). below a $T_{\min }$ in the range between 30 and $40 \mathrm{~K}$. The Hall mobility of LAO/ETO/STO samples in Fig. 4(b) shows the characteristic power-law temperature dependence similar to standard LAO/STO as a consequence of scattering by optical phonons [23]. However, at low temperatures we observe a downturn below $T_{\min }$ corresponding to the upturn of the sheet resistance. Thus, the transport characterization indicates that the ETO layer suppresses the conductance and the mobility of the interface, introducing another source of scattering into the system.

In order to understand whether the presence of an additional interface or the particular deposition conditions used could have a role in the enhanced scattering, we realized LAO(10)/STO(2)/STO heterostructures, with a 2-uc STO film grown under the same conditions replacing ETO. The choice of STO was driven by the negligible differences between the lattice parameters of ETO and STO, while any other nonmagnetic oxide replacing ETO would introduce another degree of freedom, which is epitaxial strain. As a matter of fact, we find that LAO/STO/STO heterostructures show transport properties similar to those of standard LAO/STO [Fig. 4(a); filled (blue) circles]. While this experiment cannot fully exclude the possibility of enhanced scattering due to the presence of an additional interface in the LAO/ETO/STO case, it suggests a specific role of ETO in the transport properties of these heterostructures and shows that the growth conditions, quite different from those usually employed to deposit LAO/STO interfaces, are not the cause for the observed results.

The upturn of the low-temperature resistivity and the simultaneous mobility suppression, shown in Fig. 4, were already observed in LAO/STO [2,5] and STO [24], depending on carrier density and on deposition conditions. This behavior was interpreted as a signatures of weak [2] or strong localization of carriers at low temperatures or as a signature of Kondo scattering [5,24]. In general, in localization models, the resistance vs temperature is characterized by a positive curvature down to the lowest temperature, with no signature of saturation. Kondo scattering, on the other hand, is characterized by a change in the curvature of the resistance $\left(d^{2} R / d T^{2}\right)$ from positive (no saturation) to negative (saturation effect) at low temperatures. As shown in detail in the Supplementary Material [21], the temperature dependence of the resistivity is characterized by a change in the curvature below $10 \mathrm{~K}$ so that the $d^{2} R / d T^{2}$ goes from positive to negative values. Moreover, any attempt to fit data within a weak [25] (or strong) localization framework fails. Thus we propose that the resistivity upturn is due to Kondo-like magnetic scattering of the Q2DES. To verify the consistency of this hypothesis, we fit the transport data taking into account the presence of Kondo scattering. The complete formula used to fit the sheet resistance curves is assumed to be the sum of four terms:

$$
R(T)=R_{0}+R_{K}(T)+A T^{2}+R_{\mathrm{BG}}(T) .
$$

$R_{0}$ is the temperature independent term due to nonmagnetic impurity scattering, the $T^{2}$ contribution is a consequence of a quasinonretarded interaction between dressed quasiparticles in STO [26], $R_{\mathrm{BG}}(T)$ is the phonon scattering term described by the Bloch-Gruneisen law, and $E_{K}(T)$ is the Kondo term related to scattering by magnetic impurities. For $R_{K}(T)$ we 
use the zero-field generalized Hamann expression [27]:

$$
R_{K}(T)=C\left(1-\frac{\ln \left(T / T_{K}\right)}{\sqrt{\left(\ln \left(T / T_{K}\right)\right)^{2}+\pi^{2}(S(S+1))}}\right) .
$$

Here, $\mathrm{T}_{K}$ is the effective Kondo temperature and $S$ is the effective spin of the magnetic scattering centers. We get a consistent fit [Fig. 4(a); solid black lines] using the Kondo model for all the LAO/ETO/STO heterostructures. In particular, we find a Kondo temperature which varies slightly around $T_{K} \sim 10 \mathrm{~K}$, while the parameter $S$ is always found to be close to $S=0.22$, in good agreement with the value expected from numerical renormalization group theory for a Kondo impurity with spin $1 / 2$ and similar to experimental values obtained for other titanates [28].

We also investigated the magnetoresistance as function of the temperature of LAO/ETO/STO samples up to a field of $9 \mathrm{~T}$ perpendicular to the interface (see Fig. S4 in the Supplementary Material [21]). The dominant contribution to the magnetoresistance is classical cyclotronic magnetic field dependence $\left(R \sim H^{2}\right)$. Some deviations are seen at low temperatures $(<10 \mathrm{~K})$ and low fields $(<3-4 \mathrm{~T})$, where spin-orbit scattering and weak antilocalization start to play an important role. Interestingly, the simultaneous presence of different scattering contributions, including spin-orbit scattering and classical cyclotronic terms, were also observed in the case of liquid gated STO [24], which shows Kondo transport behavior. Thus these data are compatible with the presence of Kondo scattering in our samples.

\section{DISCUSSION}

The transport properties of LAO/ETO/STO suggest the simultaneous presence of different scattering mechanisms contributing to the transport properties. These include classical elastic scattering, spin-orbit scattering, and Kondo scattering. Kondo scattering is the main scattering contribution at low temperatures as suggested by the characteristic saturation of the resistivity and by the consistent fit of the data shown in Fig. 4. However, spin-orbit scattering competes with Kondo scattering at low temperatures, as shown by magnetoconductance data. Due to multiple scattering channels, the role of ETO ferromagnetism in the transport properties of the Q2DES is evidently hidden. Indeed, for the range of carrier densities investigated, the Q2DES does not show a ferromagnetic character, which would manifest in a metallic low-temperature transport and, eventually, a downturn in the resistivity at the ETO ferromagnetic transition.

To interpret these results, we start from a polar catastrophe scenario, where electrons are expected to move first to the ETO layers. The large energetic cost associated with on-site Coulomb repulsion of two electrons at $4 f$ sites $(U=6 \mathrm{eV}$ from Ref. [14]) hinders the transfer of electrons to the $\mathrm{Eu}$ levels. Thus we expect a filling of the Ti-3d states belonging to ETO. However, the transport data suggest that the Q2DES has a strong contribution from electrons transferred to STO itself. Indeed, as long as the ETO film is thin enough, at least a fraction of the doping electrons could leak also into STO at the interface, leading to the formation of a Q2DES as in standard LAO/STO. On the other hand, when the ETO thickness exceeds 2 uc, all the electrons remain trapped in the ETO layer and apparently do not contribute to the formation of a Q2DES extending in the underneath STO. According to this picture, the transport properties of LAO/ETO/STO are essentially those of $\mathrm{LAO/STO}$, with the addition of a ferromagnetic layer (ETO) in close proximity to the Q2DEG. This scenario accounts for the observation that the mobile charge carrier density, the sheet resistance, and the overall characteristics of LAO/ETO/STO interfaces are similar to those of standard LAO/STO interfaces. It remains to be explained why the electrons transferred to an ETO a few unit cells thick (3-6) are not mobile. One possibility is that electron correlations, possibly favored by a partial overlapping of Ti-3 $d$ and Eu- $4 f$ unoccupied states [14], play an important role as shown in other $\delta$-doped heterostructures [13].

However, the enhanced low-temperature scattering of magnetic origin suggests that the Q2DES may extend also in the ETO layer and eventually couple to the magnetic ions in the system. We cannot establish at the moment the origin of the Kondo impurity scattering centers. Possible candidates are excess electrons transferred to localized titanium states at the interface, acting as dilute magnetic scattering centers (at the ETO/STO interface or in the ETO film), or $\mathrm{Eu}^{2+}$ magnetic scattering centers for the electrons in the ETO layer itself. On the other hand, we can probably exclude any role of $\mathrm{Eu}^{2+} / \mathrm{Sr}^{2+}$ substitution in $\mathrm{SrTiO}_{3}$, since the ferromagnetic character of ETO ultrathin films is hardly reconciled with a disordered intermixed $\mathrm{Eu}_{1-x} \mathrm{Sr}_{x} \mathrm{TiO}_{3}$ layer at the interface [29]. According to this picture, the $\delta$-doped ETO layer has a fundamental role in controlling the transport properties of the LAO/STO interface by introducing localized magnetic scattering centers. In this Kondo scenario the Q2DES screens the magnetic scattering centers, which suggests the presence of a negative exchange interaction between mobile electrons and localized spins.

\section{CONCLUSIONS}

In conclusion, we have grown high-quality LAO/ETO/STO heterostructures using RHEED-assisted pulsed laser deposition. This system shows a Q2DES when the ferromagnetic ETO layer is below 3 uc. While transport data do not allow us to firmly establish a magnetic coupling between the Q2DES and the magnetism of ETO, these results show that $\delta$ doping by magnetic layers is a viable route to engineering the properties of the Q2DES in LAO/STO heterostructures. Further experimental investigations are needed to establish whether or not, and under what conditions (e.g., doping level), the Q2DES in LAO/ETO/STO heterostructures can be made spin polarized.

\section{ACKNOWLEDGMENTS}

The authors acknowledge C. A. Perroni and P. Lucignano for valuable discussions. We received funding from the Ministero dell'Istruzione, dell'Università e della Ricerca (Grant No. PRIN 2010-11-OXIDE), and the FIRB 2012 project HybridNanoDev (Grant No. RBFR1236VV). 
[1] A. Ohtomo and H. Y. Hwang, Nature 427, 423 (2004).

[2] A. D. Caviglia, S. Gariglio, C. Cancellieri, B. Sacépé, A. Fête, N. Reyren, M. Gabay, A. F. Morpurgo, and J.-M. Triscone, Phys. Rev. Lett. 105, 236802 (2010).

[3] S. Thiel et al., Science 313, 1942 (2006).

[4] N. Reyren, S. Thiel, A. D. Caviglia, L. Fitting Kourkoutis, G. Hammerl, C. Richter, C. W. Schneider, T. Kopp, A. S. Ruetschi, D. Jaccard, M. Gabay, D.-A. Muller, J.-M. Triscone, and J. Mannhart, Science 317, 1196 (2007).

[5] A. Brinkman et al., Nature Mater. 6, 493 (2007).

[6] L. Li, C. Richter, J. Mannhart, and R. C. Ashoori, Nature Phys. 7, 762 (2011).

[7] J. A. Bert, B. Kalisky, C. Bell, M. Kim, Y. Hikita, H. Y. Hwang, and K. A. Moler, Nature Phys. 7, 767 (2011).

[8] N. Reyren, M. Bibes, E. Lesne, J. M. George, C. Deranlot, S. Collin, A. Barthelemy, and H. Jaffres, Phys. Rev. Lett. 108, 186802 (2012).

[9] W. Han, X. Jiang, A. Kajdos, S.-H. Yang, S. Stemmer, and S. S. P. Parkin, Nat. Commun. 4, 2134 (2013).

[10] M. Salluzzo, S. Gariglio, D. Stornaiuolo, V. Sessi, S. Rusponi, C. Piamonteze, G. M. De Luca, M. Minola, D. Marre, A. Gadaleta, H. Brune, F. Nolting, N. B. Brookes, and G. Ghiringhelli, Phys. Rev. Lett. 111, 087204 (2013).

[11] T. Fix, F. Schoofs, J. L. MacManus-Driscoll, and M. G. Blamire, Appl. Phys. Lett. 97, 072110 (2010).

[12] S. Das, A. Rastogi, L. Wu, J.-C. Zheng, Z. Hossain, Y. Zhu, and R. C. Budhani, arXiv:1402.1915 [cond-mat].

[13] H. W. Jang, D. A. Felker, C. W. Bark, Y. Wang, M. K. Niranjan, C. T. Nelson, Y. Zhang, D. Su, C. M. Folkman, S. H. Baek, S. Lee, K. Janicka, Y. Zhu, X. Q. Pan, D. D. Fong, E. Y. Tsymbal, M. S. Rzchowski, and C. B. Eom, Science 331, 886 (2011).

[14] R. Ranjan, H. Sadat Nabi, and R. Pentcheva, J. Phys.: Condens. Matter 19, 406217 (2007).

[15] T. Katsufuji and Y. Tokura, Phys. Rev. B 60, R15021 (1999).
[16] J. H. Lee, L. Fang, E. Vlahos, X. Ke, Y. W. Jung, L. F. Kourkoutis, J.-W. Kim, P. J. Ryan, T. Heeg, M. Roeckerath, V. Goian, M. Bernhagen, R. Uecker, P. C. Hammel, K. M. Rabe, S. Kambal, J. Schubert, J. W. Freeland, D. A. Muller, C. J. Fennie, P. Schiffer, V. Gopalan, E. Johnston-Halperin, and D. G. Schlom, Nature 466, 954 (2010).

[17] C. Piamonteze, U. Flechsig, S. Rusponi, J. Dreiser, J. Heidler, M. Schmidt, R. Wetter, M. Calvi, T. Schmidt, H. Pruchova, J. Krempasky, C. Quitmann, H. Brune, and F. Nolting, J. Synchrotron Radiat. 19, 661 (2012).

[18] N. L. Henderson, J. Baek, P. S. Halasyamani, and R. E. Schaak, Chem. Mater. 19, 1883 (2007).

[19] E. Stavitski and F. M. F. de Groot, Micron 41, 687 (2010).

[20] The relationship between the expectation value of the effective spin moment operator and that of the spin moment operator is, in the case of $4 f$ rare earth, $\left\langle S_{E z}\right\rangle=\left\langle S_{z}\right\rangle+3\left\langle T_{z}\right\rangle$, where $T_{z}$ is the quadrupole moment. $\left\langle T_{z}\right\rangle$ is, in general, much smaller than $\left\langle S_{z}\right\rangle$ and has been neglected in this case.

[21] See Supplemental Material at http://link.aps.org/supplemental/ 10.1103/PhysRevB.89.224413 for more details.

[22] K. Fujita, N. Wakasugi, S. Murai, Y. Zong, and K. Tanaka, Appl. Phys. Lett. 94, 062512 (2009).

[23] O. N. Tufte and P. W. Chapman, Phys. Rev. 155, 796 (1967).

[24] M. Lee, J. R. Williams, S. Zhang, C. D. Frisbie, and D. Goldhaber-Gordon, Phys. Rev. Lett. 107, 256601 (2011).

[25] P. A. Lee and T. V. Ramakrishnan, Rev. Mod. Phys. 57, 287 (1985).

[26] D. van der Marel, J. L. M. van Mechelen, and I. I. Mazin, Phys. Rev. B 84, 205111 (2011).

[27] D. R. Hamann, Phys. Rev. B 158, 570 (1967).

[28] R. Ramaneti, J. C. Lodder, and R. Jansen, Phys. Rev. B 76, 195207 (2007).

[29] Z. Guguchia, H. Keller, A. Bussmann-Holder, J. Kohler, and R. K. Kremer, Eur. Phys. J. B 86, 409 (2013). 